\title{
Desempenho visual na correção de miopia com óculos e lentes de contato gelatinosas
}

\author{
Visual performance in myopic correction with \\ spectacles and soft contact lenses
}

\author{
Breno Barth ${ }^{1}$ \\ Mílton Ruiz Alves ${ }^{2}$ \\ Newton Kara-José ${ }^{3}$
}

Trabalho realizado na Faculdade de Medicina da Universidade de São Paulo e Eye Clinic Day Hospital - São Paulo (SP) - Brasil.

${ }^{1}$ Doutor pela Faculdade de Medicina da Universidade de São Paulo - USP - São Paulo (SP) - Brasil.

${ }_{2}^{2}$ Professor Livre Docente da Universidade de Campinas - UNICAMP - Campinas (SP) - Brasil e da USP - São Paulo (SP) - Brasil

${ }^{3}$ Professor Titular da USP - São Paulo (SP) - Brasil Professor Titular da UNICAMP - Campinas (SP) - Brasil.

Endereço para correspondência: Breno Barth. Rua Miguel Rocha, 1920 - Apto. 800 - Natal (RN)

CEP 59064-580

E-mail: brenobarth@digizap.com.br

Recebido para publicação em 09.10.2006

Última versão recebida em 28.09.2007

Aprovação em 29.09.2007

Os autores não possuem interesse financeiro no desenvolvimento ou marketing das medicações ou instrumentos referidos no estudo.

Nota Editorial: Depois de concluída a análise do artigo sob sigilo editorial e com a anuência do Dr. Marcelo Carvalho da Cunha sobre a divulgação de seu nome como revisor, agradecemos sua participação neste processo.

\section{RESUMO}

Objetivos: Avaliar o desempenho visual, por meio da acuidade visual LogMAR de alto contraste, sensibilidade ao contraste e análise de frentes de onda de indivíduos portadores de miopia com ou sem astigmatismo, corrigidos com óculos e com três diferentes lentes de contato gelatinosas [Acuvue ${ }^{\circledR} 2$ (Vistacon J\&J Vision Care Inc., EUA), Biomedics ${ }^{\circledR} 55$ (Ocular Science, EUA) e Focus ${ }^{\circledR}$ 1-2 week (Ciba Vision Corporation, EUA)]. Métodos: Estudo clínico e prospectivo com intervenção em amostra constituída por quarenta indivíduos portadores de miopia entre $-1,00 \mathrm{D}$ e -4,50 D, sem ou com astigmatismo até - 0,75 D. Resultados: A sobrerefração objetiva realizada pelo aberrômetro Zywave detectou diferença significativa entre as lentes de contato gelatinosas Acuvue ${ }^{\circledR} 2$ e Biomedics ${ }^{\circledR}$ 55 , em relação aos componentes refrativos esfera e equivalente esférico, que se mostraram hipercorrigidos em relação às lentes de contato gelatinosas Focus ${ }^{\circledR}$ 1-2 week. A acuidade visual LogMAR e sensibilidade ao contraste não detectaram diferenças no desempenho visual com os óculos e com as três lentes de contato gelatinosas. A análise de frentes de onda detectou diferença significativa na aberração de terceira ordem sem e com as lentes de contato gelatinosas, com melhor desempenho visual com as lentes de contato gelatinosas Acuvue ${ }^{\circledR} 2$ e Biomedics ${ }^{\circledR} 55$. Conclusões: A análise de frentes de onda detectou diferenças no desempenho visual com óculos e com as lentes de contato gelatinosas, sendo, portanto, uma medida mais sensível da função visual que a acuidade visual LogMAR de alto contraste e a sensibilidade ao contraste. O modelo de avaliação de desempenho visual com análise de frentes de onda utilizado nesta investigação serve de modelo para pesquisas semelhantes.

Descritores: Miopia; Óculos; Lentes de contato; Acuidade visual; Sensibilidades de contraste; Estudos prospectivos

\section{INTRODUCÃO}

A correção de erros refrativos com lentes oftálmicas (óculos) altera o mundo visual do indivíduo. As imagens borradas deverão tornar-se nítidas e poderão parecer estar em distâncias distintas daquelas dos objetos originais. As imagens poderão estar aumentadas ou diminuídas, e efeitos prismáticos poderão modificar a posição aparente dos objetos. As aberrações produzidas pelas lentes oftálmicas também poderão alterar a forma e a nitidez das imagens ${ }^{(1)}$. A despeito da qualidade óptica da lente de contato (LC) ter melhorado, alguns indivíduos queixam-se da visão corrigida com LC, embora apresentem acuidade visual com a melhor correção (AVcc) de $20 / 20^{(2)}$. Vários investigadores analisaram o desempenho visual com LC e 
com óculos e verificaram diminuição de sensibilidade ao contraste com as LC em comparação com os óculos ${ }^{(3-7)}$. As LC gelatinosas (LCG) são amplamente utilizadas, mas muito pouco se conhece do desempenho visual com LCG de diferentes fabricantes. Atualmente, muito mais esforço e tempo são gastos avaliando-se alterações de acuidade visual (AV), sensibilidade ao contraste e aberrações de alta ordem em indivíduos submetidos a tratamentos fotoablativos convencionais e personalizados do que com a correção de erros refrativos com óculos e LC. A criação de um banco de dados a partir de investigações realizadas nessa área permitirá fornecer subsídios que poderão contribuir para melhorar a qualidade óptica e o desempenho visual da correção do erro refrativo com lentes oftálmicas e com LC. Este estudo foi desenhado para comparar o desempenho visual de três LCG, disponíveis comercialmente, com o de óculos, utilizando-se na avaliação, AV LogMAR de alto contraste, sensibilidade ao contraste e análise de frentes de onda.

\section{MÉTODOS}

O presente estudo foi realizado na Eye Clinic Day Hospital - São Paulo (SP), com o apoio do Laboratório de Investigações Médicas (LIM - 33) da Clínica Oftalmológica do Hospital das Clínicas da Faculdade de Medicina da Universidade de São Paulo, após a aprovação de seu protocolo pelo Comitê de Ética para Análise de Projetos de Pesquisa - CAPPesq, da Diretoria Clínica do Hospital das Clínicas e da Faculdade de Medicina da Universidade de São Paulo.

Realizou-se um estudo clínico e prospectivo com intervenção. Foram selecionados, consecutivamente, 73 indivíduos, portadores de miopia com ou sem astigmatismo, não usuários de LC, com motivação para o uso de LCG, examinados no Setor de Lentes de Contato da Eye Clinic Day Hospital, no período de $1^{\circ}$ de maio de 2003 a 30 de setembro de 2003 . Dentre os casos selecionados, 40 indivíduos, que preencheram os critérios de inclusão e exclusão, constituíram a amostra do estudo.

Critérios de inclusão: Indivíduos de ambos os sexos, portadores de miopia entre $-1,00 \mathrm{D}$ e $-4,50 \mathrm{D}$, sem ou com astigmatismo até $-0,75 \mathrm{D}$, não usuários de LC, com motivação para uso de LCG, com acuidade visual corrigida com óculos e com as LCG igual ou superior a 20/20; capacidade de compreender e seguir as orientações recomendadas de uso e manuseio das LCG; assinatura do termo de consentimento.

Critérios de exclusão: Presença de afecção ativa de natureza alérgica, inflamatória ou infecciosa, na superfície ocular e ou de anexos; valor do meridiano mais plano da córnea $>46,00$ $\mathrm{D}$ ou $<40,00 \mathrm{D}$; indivíduos que não puderam ser adaptados com os três tipos de LCG avaliadas; desconforto importante no teste de adaptação das LCG; recusa em participar do estudo.

As lentes dos óculos foram confeccionadas em policarbonato, com tratamento de superfície anti-risco e anti-reflexo. Os parâmetros das LCG estão no quadro 1 .
Os pacientes foram submetidos a exame oftálmico completo e ao final da avaliação, tiveram seus óculos prescritos. Posteriormente, retornaram para a adaptação das LCG, quando foram submetidos à medida da $\mathrm{AV} \operatorname{LogMAR}$ de alto contraste e as avaliações de sensibilidade ao contraste e das aberrações de alta ordem. Cada paciente teve um olho escolhido por sorteio para a avaliação do desempenho visual com óculos e LCG (sensibilidade ao contraste) e sem e com LCG (aberrometria). A seqüência de adaptação das três LCG foi definida previamente, por sorteio. Entre cada avaliação de desempenho visual fez-se um intervalo de vinte minutos.

A avaliação da centralização, movimentação e a sobrerefração foram executadas após vinte minutos da colocação daLCG.

Medida da acuidade visual de alto contraste: Utilizouse a tabela de AV do ETDRS (Early Treatment Diabetic Retinopathy Study - Estudo do Tratamento Precoce da Retinopatia Diabética) (Light House, New York, EUA), posicionada a uma distância de 6 metros (ou a 20 pés) do sujeito. Usaramse a mesma sala de exame e a mesma iluminação em todos os exames. A AV LogMar de alto contraste foi medida primeiro com os óculos, depois com cada LCG, em ordem definida previamente, por sorteio.

Medida de sensibilidade ao contraste: $O$ teste de sensibilidade ao contraste foi realizado com o aparelho CSV-1000 (VectorVision, Ohio, EUA) que verifica frequiências espaciais diferentes $(3,6,12$ e $18 \mathrm{cpg})$ em quatro fileiras (A, B, C, e D). $\mathrm{O}$ indivíduo foi posicionado a 2,4 $\mathrm{m}$ ( 8 pés) da unidade onde se encontrava a tabela, utilizando os óculos e depois cada uma das LCG. Mostrou-se ao paciente o primeiro círculo para que identificasse o padrão. À medida que o paciente "lia" a fila, considerava-se que o padrão encontrava-se no círculo inferior ou superior. Se o padrão não fosse visível, o indivíduo informava que ambos os círculos pareciam iguais, ou seja, com o aspecto de cinza homogêneo. A última resposta que identificava corretamente o padrão determinava o limiar de contraste para cada frequiência espacial testada.

Medida de aberrações ópticas de alta ordem: A medida das aberrações ópticas de alta ordem foram realizadas com o aberrômetro Zywave ${ }^{\circledR}$ (Bausch \& Lomb). Para a obtenção de midríase, instilou-se uma gota de fenilefrina a $10 \%$, vinte minutos antes do início do exame. Em primeiro lugar, posicionou-se o paciente para que, com a cabeça e o queixo confortavelmente apoiados, pudesse observar o alvo. Alinhou-se a pupila nos eixos "x" e "y", observando-se sua posição no monitor. Após a colocação de foco na íris, com o olho mantido aberto, pressionou-se o comando para iniciar o pré-scan. Em seguida, foram pressionados os comandos necessários para dar continuidade ao exame até a obtenção da grade e do centróide, visualizados no monitor, no centro da pupila. Novamente, pressionou-se o comando para calcular as aberrações ópticas. Foi considerado o diâmetro pupilar de $6 \mathrm{~mm}$. O exame foi repetido três vezes. Para efeito de cálculo, foram consideradas as médias dos três exames.

Para o processamento dos dados, foi construído um banco 


\begin{tabular}{|c|c|c|c|}
\hline Lentes de contato gelatinosas & Acuvue $^{\circledR} 2$ & Biomedics $^{\circledR} 55$ & Focus $^{\circledR}$ 1-2 week \\
\hline Material & Etafilcon A & Ocufilcon A & Vitifilcon A \\
\hline Porcentagem de água (\%) & 58 & 55 & 55 \\
\hline Espessura central LC de $-3,0 \mathrm{D}(\mathrm{mm})$ & 0,08 & 0,07 & 0,06 \\
\hline Diâmetro $(\mathrm{mm})$ & 14,0 & 14,2 & 14,0 \\
\hline $\operatorname{DK}\left(\mathrm{cm}^{2} / \mathrm{s}\right) \quad\left(\mathrm{O}_{2} / \mathrm{mlxmmHg}\right)$ & 28 & 28,2 & 26 \\
\hline
\end{tabular}

de dados com o software Access do Office 2000. Foram elaborados gráficos e tabelas com o Word e o Excel do Office 2000.

As análises estatísticas foram realizadas com o auxílio dos programas de computador SPSS 10.0, StatXact 4.0 e GraphPad InStat 3.05.

Análise descritiva: As variáveis contínuas foram analisadas por meio dos valores mensurados (resultados) nos pacientes demandados (unidades amostrais). As variáveis categóricas foram consideradas como fatores de estudo, os quais são Tipo de lente $\left(\mathrm{F}_{1}\right)$, Ordem $\left(\mathrm{F}_{2}\right)$, Nível de aberração $\left(\mathrm{F}_{3}\right)$, Nível de ordem $\left(\mathrm{F}_{4}\right)$, Sensibilidade ao contraste $\left(\mathrm{F}_{5}\right)$ e Polinômios de Zernike $\left(\mathrm{F}_{6}\right)$. As variáveis resposta são Sensibilidade (ciclos/grau) para o fator $F_{5}$ e Aberração $(\mu \mathrm{m})$ para os demais fatores. Os valores das respostas foram obtidos nas avaliações feitas junto aos pacientes da amostra. Para as variáveis contínuas, foi feita uma análise descritiva obtendose as médias, valores mínimo e máximo e desvio-padrão para todos os fatores, em separado e em conjunto.

Para fins de análise estatística, as medidas de acuidade visual foram calculadas em LogMAR. Os valores de sensibilidade ao contraste foram convertidos em unidades de log, conforme tabela fornecida pelo fabricante.

Análise comparativa: Para efeito de análise dos resultados da aberração e da sensibilidade, utilizou-se o planejamento experimental fatorial, onde são testados quatro modelos que envolvem a combinação dos cinco fatores do experimento com o fator 1 - Tipo de lente.

A técnica inferencial estatística apresenta os resultados dos testes de hipótese que representam os objetivos da tese. A estatística usada para testar as hipóteses foi a F de Snedcor (Fisher). Para a significância da rejeição ou não das hipóteses, usou-se o p-valor.

Para a comparação dos grupos dois a dois, foi utilizado o teste F de Snedcor e na análise de amostras independentes, a prova F de Snedcor (Fisher).

Todos os testes foram realizados na forma bicaudal e admitindo-se o nível de significância de 5\%. Níveis descritivos (p) inferiores a esse valor foram considerados significantes. Utilizou-se o programa de computador Statistica for Windows.

\section{RESULTADOS}

Os dados demográficos e aspectos clínicos dos participantes foram os seguintes: média de idade e desvio-padrão, respectivamente, $24,41 \pm 4,49$ anos; $45 \%$ do sexo masculino e $55 \%$ do sexo feminino, cada um dos olhos foi sorteado em metade das vezes, e equivalente esférico do erro refrativo e desvio-padrão, respectivamente, $-2,33 \pm 0,81 \mathrm{D}$. Todos os indivíduos apresentaram a AV LogMAR de 0,00, com os óculos e com cada uma das três LCG.

Os valores de sensibilidade ao contraste e respectivas freqüências espaciais, com os óculos e com as LCG, estão na tabela 1 e figura 1.

Os valores de sensibilidade ao contraste não foram influenciados pela correção dos erros refrativos com os óculos ou com as LCG.

Os resultados das aberrações de alta ordem representadas pelos polinômios de Zernike, para o diâmetro pupilar de $6 \mathrm{~mm}$, encontram-se na tabela 2.

Houve diferença significativa nos polinômios Z331, Z330, Z440, Z511 e Z551. Os resultados do emprego do teste de contraste envolvendo as médias, duas a duas, em ordem decrescente de RMS, mostraram que:

- Polinômio Z331: $\left(\right.$ Acuvue $^{\circledR} 2=$ Biomedics $^{\circledR} 55$ ) \# sem LC \# Focus ${ }^{\circledR}$ 1-2 week;

- Polinômios Z330 e Z440: $\left(\right.$ Acuvue $^{\circledR} 2=$ Biomedics $^{\circledR} 55=$ Focus $^{\circledR}$ 1-2 week) \# sem LC;

- Polinômio Z511: (Biomedics ${ }^{\circledR} 55=$ sem LC) \# $\left(\right.$ Focus $^{\circledR} 1-2$ week $=$ Acuvue $\left.^{\circledR} 2\right)$;

- Polinômio Z551: (Acuvue ${ }^{\circledR} 2=\operatorname{sem}$ LC) \# $\left(\right.$ Biomedics $^{\circledR} 55=$ Focus ${ }^{\circledR}$ 1-2 week).

Os valores das aberrações de alta ordem em RMS, para o diâmetro pupilar de 6 mm, estão na tabela 3 .

Houve diferença significativa na aberração de terceira ordem. Os resultados do emprego do teste de contraste envolvendo as médias, duas a duas, em ordem decrescente de RMS, mostraram que:

- $\left(\right.$ Acuvue ${ }^{\circledR} 2=$ Biomedics $\left.^{\circledR} 55\right)$ \# (Focus ${ }^{\circledR} 1-2$ week = sem LC).

$\mathrm{Na}$ figura 2, está a representação gráfica dos valores de RMS da aberração de terceira ordem.

Os valores dos níveis das aberrações de alta ordem sem e com as LCG, para o diâmetro pupilar de $6 \mathrm{~mm}$, estão na tabela 4 .

Houve diferença significativa no nível de aberração trifoil. Os resultados do emprego do teste de contraste envolvendo as médias, duas a duas, em ordem decrescente de RMS, mostraram que:

- $\left(\right.$ Acuvue $^{\circledR} 2=$ Biomedics $^{\circledR} 55$ ) \# Focus ${ }^{\circledR}$ 1-2 week \# sem LC.

Na figura 3, está a representação gráfica dos valores de RMS da aberração trifoil. 


\section{DISCUSSÃO}

Não houve diferença na AV LogMAR de alto contraste entre os óculos e as três LCG. O critério de inclusão no estudo selecionou indivíduos portadores de miopia e astigmatismo até - $0,75 \mathrm{D}$ com AV cc de 20/20 (Snellen). Investigações anteriores, mostraram que a correção de erros refrativos com óculos ou com LCG não apresentava diferenças de desempenho visual, quando avaliado pela AV LogMar cc de alto contraste, sugerindo que o desempenho visual deveria ser avaliado pela sensibilidade ao contraste ${ }^{(7-8)}$. A sensibilidade ao contraste é uma medida mais sensível da função visual que a AV LogMAR de alto contraste, não somente na avaliação com LC, mas também em vários estados de doença ocular ${ }^{(8-10)}$. No entanto, neste estudo, a avaliação de sensibilidade ao contraste não identificou diferenças entre os óculos e as três LCG (Tabela 1 e Figura 1). Tais resultados diferem de autores que encontraram redução de sensibilidade ao contraste com LCG em comparação com os óculos ${ }^{(7)}$. Diferem, também, de estudos que encontraram redução significativa de sensibilidade ao contraste, na frequiência de 12 ciclos por grau, quando compararam a LC Cibasoft com os óculos ${ }^{(8)}$. A redução de sensibilidade ao contraste em freqüências mais baixas foi igualmente observada com LCG com baixo conteúdo de água ${ }^{(11)}$ e com maior espessura central ${ }^{(12)}$. Embora, neste estudo, as LCG sejam de fabricantes e de materiais diferentes (Quadro 1), não

\begin{tabular}{|c|c|c|c|c|c|c|}
\hline Freqüência espacial & Óculos & \multicolumn{3}{|c|}{ Lentes de contato } & \multicolumn{2}{|c|}{ Teste } \\
\hline Freqüência 3 & $1,904 \pm 0,151$ & $1,911 \pm 0,137$ & $1,915 \pm 0,147$ & $1,904 \pm 0,161$ & 0,054 & 0,9834 \\
\hline Freqüência 6 & $2,151 \pm 0,129$ & $2,174 \pm 0,120$ & $2,174 \pm 0,129$ & $2,151 \pm 0,160$ & 0,364 & 0,7790 \\
\hline Freqüência 12 & $1,814 \pm 0,134$ & $1,836 \pm 0,120$ & $1,814 \pm 0,154$ & $1,807 \pm 0,145$ & 0,312 & 0,7951 \\
\hline
\end{tabular}

Tabela 2. Aberrações de alta ordem representadas pelos polinômios de Zernike em RMS em micrômetros sem e com as lentes de contato gelatinosas (Acuvue ${ }^{\oplus}$ 2, Biomedics ${ }^{\circledast} 55$ e Focus $^{\oplus}$ 1-2 week). Eye Clinic Day Hospital, São Paulo - 2003

\begin{tabular}{|c|c|c|c|c|c|c|c|c|}
\hline \multirow{2}{*}{$\begin{array}{l}\text { Polinômios de } \\
\text { Zernike }\end{array}$} & \multicolumn{8}{|c|}{$\mathrm{N}=40$} \\
\hline & Sem LC & \multicolumn{5}{|c|}{ Lentes de contato } & \multicolumn{2}{|c|}{ Teste } \\
\hline Z311 & $0,142 \pm 0,071$ & 0,180 & $\pm 0,103$ & 0,150 & $\pm 0,119$ & $\begin{array}{cc}\text { Focus }^{\circledR} & \mathbf{1 - 2} \text { week } \\
0,177 & \pm 0,102\end{array}$ & $\begin{array}{c}\mathbf{F} \\
1,44\end{array}$ & $\begin{array}{c}\mathbf{P} \\
0,2327\end{array}$ \\
\hline Z331 & $0,146 \pm 0,113$ & 0,079 & $\pm 0,055$ & 0,074 & $\pm 0,044$ & $0,169 \pm 0,094$ & 13,65 & 0,0000 \\
\hline Z330 & $0,128 \pm 0,127$ & 0,073 & $\pm 0,051$ & 0,087 & $\pm 0,075$ & $0,093 \pm 0,067$ & 2,94 & 0,0349 \\
\hline Z400 & $0,083 \pm 0,059$ & 0,1007 & $\pm 0,073$ & 0,110 & $\pm 0,055$ & $0,0807 \pm 0,060$ & 2,07 & $0,106 \mathrm{c}$ \\
\hline Z440 & $0,052 \pm 0,033$ & 0,0376 & $\pm 0,026$ & 0,0322 & $\pm 0,030$ & $0,0411 \pm 0,035$ & 2,94 & 0,0349 \\
\hline Z441 & $0,0362 \pm 0,022$ & 0,0385 & $\pm 0,026$ & 0,0344 & $\pm 0,026$ & $0,0483 \pm 0,027$ & 2,36 & 0,0732 \\
\hline Z510 & $0,023 \pm 0,020$ & 0,02037 & $7 \pm 0,018$ & 0,0204 & $\pm 0,015$ & $0,0180 \pm 0,014$ & 0,57 & 0,6336 \\
\hline Z511 & $0,0227 \pm 0,018$ & 0,0311 & $\pm 0,022$ & 0,0185 & $\pm 0,017$ & $0,0280 \pm 0,023$ & 3,04 & 0,0309 \\
\hline Z530 & $0,0180 \pm 0,014$ & 0,0142 & $\pm 0,011$ & 0,0140 & $5 \pm 0,012$ & $0,0171 \pm 0,022$ & 0,70 & 0,5542 \\
\hline
\end{tabular}

Tabela 3. Aberrações de alta ordem em RMS em micrômetros, sem e com as lentes de contato gelatinosas (Acuvue ${ }^{\circledR}$ 2, Biomedics ${ }^{\oplus} 55$ e Focus ${ }^{\circledR}$ 1-2 week). Eye Clinic Day Hospital, São Paulo - 2003

\begin{tabular}{|c|c|c|c|c|c|c|}
\hline Ordem & \multicolumn{4}{|c|}{$\mathrm{N}=\mathbf{4 0}$} & \multicolumn{2}{|c|}{ Teste } \\
\hline Terceira & $0,302 \pm 0,168$ & $0,254 \pm 0,084$ & $0,243 \pm 0,060$ & $0,306 \pm 0,020$ & 3,637 & 0,029 \\
\hline Quinta & $0,063 \pm 0,130$ & $0,062 \pm 0,116$ & $0,058 \pm 0,049$ & $0,066 \pm 0,034$ & 1,038 & 0,357 \\
\hline
\end{tabular}


foi possível, por meio da medida de sensibilidade ao contraste, identificar diferenças no desempenho visual entre elas e os óculos (Tabela 1 e Figura 1).

$\mathrm{Na}$ tabela 2, as aberrações ópticas de alta ordem estão representadas pelos polinômios de Zernike, que são uma maneira de se caracterizar e quantificar superfícies permitindo a sua comparação. Em relação aos coeficientes de terceira ordem, ocorreu diferença significativa nos polinômios Z330 e Z331 que representam, conjuntamente, a aberração trifoil. Em relação ao coeficiente Z330 não houve diferença significativa no desempenho visual entre as três LCG, que foi superior ao observado sem LC. Em relação ao polinômio Z331, não houve diferença significativa no desempenho visual entre as LCG Acuvue ${ }^{\circledR} 2$ e Biomedics $^{\circledR} 55$, que foi superior àquele sem LC e com a LCG Focus ${ }^{\circledR}$ 1-2 week. Em relação ao polinômio Z440, não houve diferença significativa no desempenho visual entre as três LCG, o qual foi superior ao sem LC. Em relação aos coeficientes de quinta ordem, considerando-se o polinômio Z511, não houve diferença significativa no desempenho visual entre as LCG

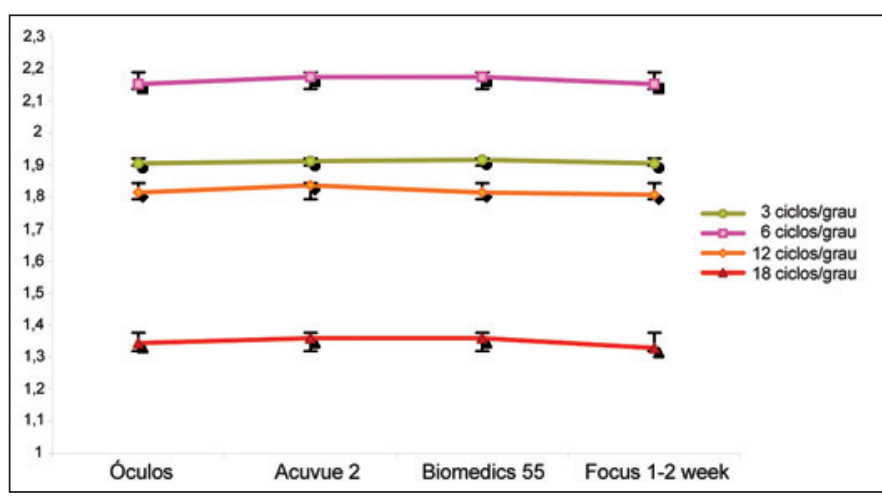

Figura 1 - Representação gráfica dos valores de sensibilidade ao contraste em unidades logarítmicas nas freqüências $3,6,12$ e 18 ciclos por grau com os óculos e com as lentes de contato gelatinosas (Acuvue $^{\circledast}$ 2, Biomedics ${ }^{\circledR} 55$ e Focus $^{\circledast}$ 1-2 week). Eye Clinic Day Hospital, São Paulo - 2003.

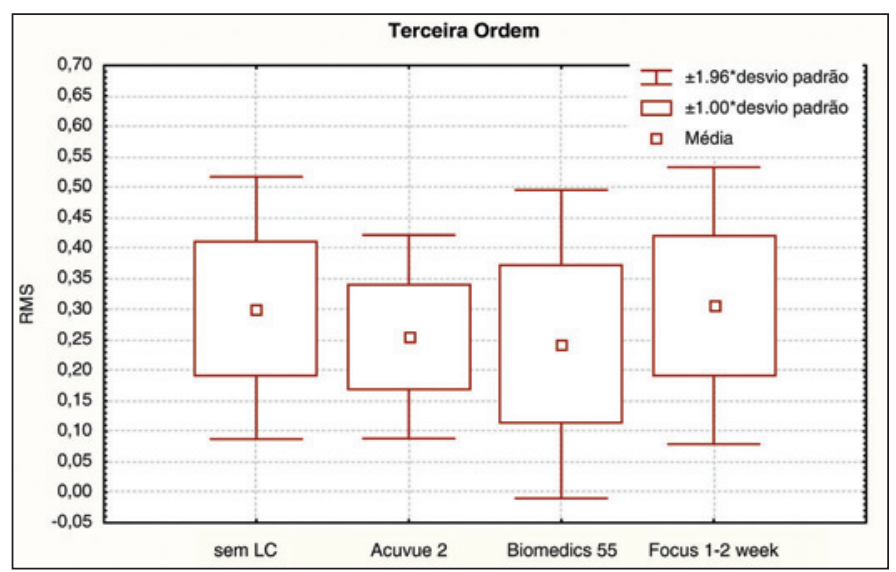

Figura 2 - Representação gráfica dos valores de RMS em micrômetros da aberração de terceira ordem sem e com as lentes de contato gelatinosas (Acuvue ${ }^{\circledR}$ 2, Biomedics ${ }^{\circledR} 55$ e Focus $^{\circledR}$ 1-2 week). Eye Clinic Day Hospital, São Paulo - 2003.
Biomedics $^{\circledR} 55$ e sem LC, que foi superior ao observado com as LCG Focus ${ }^{\circledR}$ 1-2 week e Acuvue ${ }^{\circledR} 2$ e, em relação ao polinômio Z551, não houve diferença significativa no desempenho visual entre as LCG Acuvue ${ }^{\circledR} 2$ e sem LC, que foi superior ao observado com as LCG Biomedics ${ }^{\circledR} 55$ e Focus ${ }^{\circledast}$ 1-2 week.

Os resultados das aberrações de alta ordem estão na tabela 3 e na figura 2. Houve diferença significativa na aberração de terceira ordem sem e com as LCG. Não houve diferença entre o desempenho visual com as LCG Acuvue ${ }^{\circledR} 2$ e Biomedics ${ }^{\circledR} 55$, o qual foi superior ao observado com a LCG Focus ${ }^{\circledR} 1-2$ week e sem LCG. Observou-se que, em termos individuais, a maior média absoluta dos valores de RMS foi a dos níveis de aberração coma, trifoil e tetrafoil (Tabela 4). Esta ordem de distribuição dos resultados foi concordante com a encontrada por autores, em avaliação realizada em 532 olhos de 306 indivíduos normais ${ }^{(13)}$. As aberrações de alta ordem representam o agrupamento das aberrações de coma, trifoil, aberração esférica, astigmatismo secundário, tetrafoil, coma secundário, trifoil secundário e pentafoil. A análise comparativa da distribuição dessas aberrações entre os olhos sem LC e com LCG mostrou diferença estatisticamente significativa no nível trifoil (Tabela 4 e Figura 3). Em relação ao componente trifoil, o teste de contraste comparando as médias, duas a duas, mostrou maior nível de aberração sem LCG, e em ordem decrescente de RMS, LCG Focus ${ }^{\circledR}$ 1-2 week, Biomedics ${ }^{\circledR} 55$ e Acuvue $^{\circledR} 2$. Os níveis de aberração que se situam mais ao centro da pirâmide de Zernike afetam mais a qualidade visual do que aqueles que se situam mais próximos da periferia da pirâmide ${ }^{(14)}$. Níveis diferentes de aberração com o mesmo valor de RMS influenciam de forma distinta o desempenho visual ${ }^{(14)}$.

As aberrações ópticas, neste estudo, foram medidas com o aberrômetro Zywave ${ }^{\circledast}$ que utiliza o sensor de Hartmann-Shack, o qual mede as aberrações oculares totais. Outros videoceratoscópios computadorizados podem medir a forma corneana, desenhar mapas de elevação e realizar a decomposição polinomial que é utilizada para determinar as aberrações cornea-

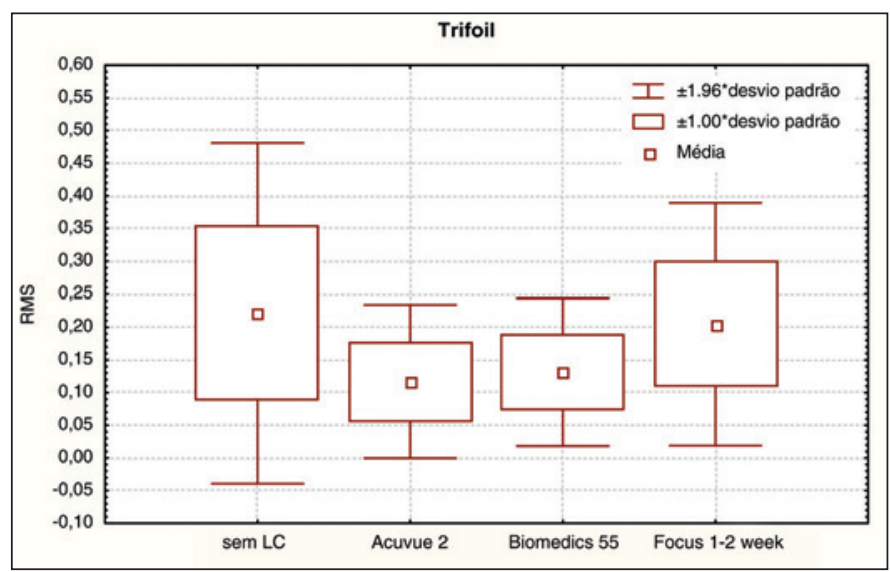

Figura 3 - Representação gráfica dos valores de RMS em micrômetros da aberração trifoil sem e com as lentes de contato gelatinosas (Acuvue ${ }^{\circledR}$ 2, Biomedics ${ }^{\circledR} 55$ e Focus ${ }^{\circledR}$ 1-2 week). Eye Clinic Day Hospital, São Paulo - 2003 


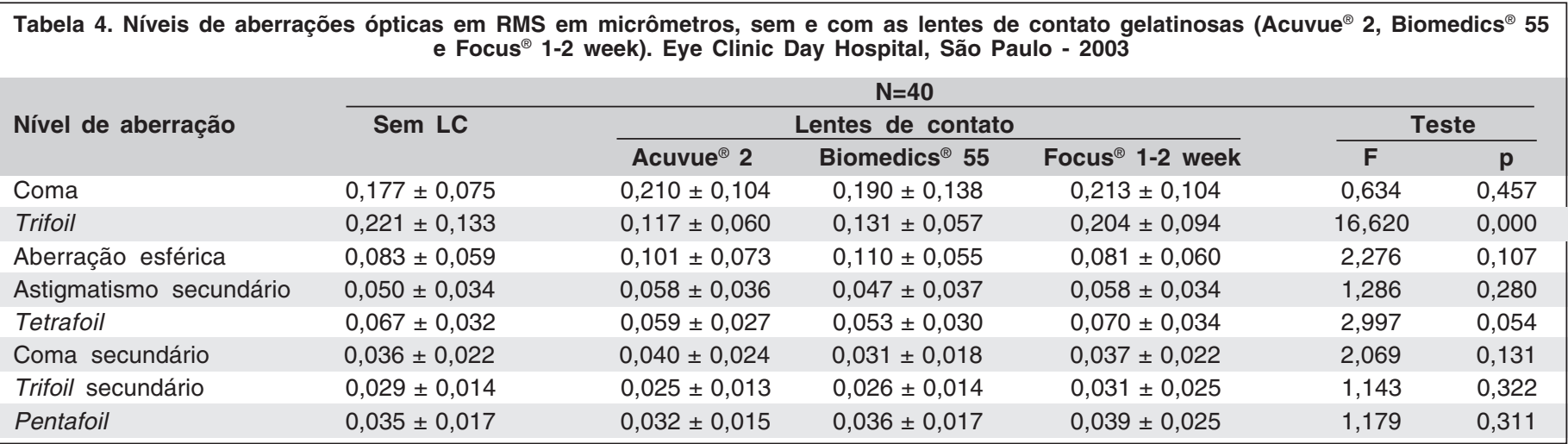

nas $^{(15-16)}$. O conhecimento das aberrações de frentes de onda corneana e lenticular é essencial para o entendimento da natureza de cada aberração e para sua correção. Esse conhecimento é fundamental para se obter LC, de forma personalizada, para a correção de aberrações ópticas de alta ordem. $\mathrm{Na}$ prática, existem inúmeras limitações para a correção com êxito de aberrações de alta ordem com LC. A centralização, movimentação e rotação das LC dificultam a correção de aberrações de alta ordem. Para a correção de aberrações de alta ordem com LC, são necessários alinhamento e estabilidade rotacional mais acurados que os requeridos para se obter bom desempenho visual com LC tórica. Qualquer movimento da LC durante o piscar ou rotação ocular diminui a efetividade da correção óptica. Com os desenhos atuais das LC, ainda não é possível conseguir a estabilidade necessária para a correção efetiva de aberrações de alta ordem ${ }^{(17-18)}$.

A escolha dos indivíduos participantes do estudo é fundamental para garantir que os resultados representem, fielmente, o que ocorre na população de interesse. Nesta investigação, selecionou-se uma amostra de pessoas viáveis de serem estudadas, em termos de tempo e custos financeiros, e que fosse, ao mesmo tempo, grande o suficiente para controlar o erro amostral na generalização dos achados e representativa para controlar o erro sistemático dessas inferências. Para o cálculo do erro amostral para a amostra de quarenta indivíduos, utilizou-se a técnica de amostragem aleatória para população finita com variáveis contínuas (sensibilidade ao contraste e aberrações de alta ordem). Os desvios-padrão dessas duas variáveis foram, respectivamente, 0,603 e 0,326. Os desvios-padrão foram calculados para a amostra submetida a medidas repetidas. O valor calculado do erro amostral foi de 0,03. Todos os indivíduos foram submetidos a medidas repetidas de AV LogMAR de alto contraste, sensibilidade ao contraste e de aberrações de alta ordem, evitando-se, assim, a composição de grupos, eliminando-se a variação interindivíduos e, dessa forma, otimizando-se o tamanho da amostra. Para controlar o erro sistemático decorrente de inferências, utilizou-se um modelo fatorial para identificar os efeitos da correção dos erros refrativos com óculos e sem e com as três LCG sobre as respostas de sensibilidade ao contraste e de aberrações de alta de ordem. A generalização dos resultados fica, portanto, restrita a uma população de indivíduos com características semelhantes às daqueles que compuseram a amostra, que serviu de base para este estudo.

\section{CONCLUSÃO}

Na avaliação do desempenho visual com óculos e LCG, a análise de frentes de onda mostrou ser uma medida mais sensível da função visual que a AVLogMAR de alto contraste e a sensibilidade ao contraste.

O modelo de avaliação de desempenho visual utilizado nesta investigação serve de modelo para pesquisas semelhantes.

\section{ABSTRACT}

Purpose: To evaluate the visual performance by high contrast visual acuity, contrast sensitivity and wavefront in myopic patients with or without astigmatism corrected with spectacles and with three different soft contact lenses [Acuvue ${ }^{\circledR} 2$ (Vistacon J\&J Vision Care Inc., USA), Biomedics ${ }^{\circledR} 55$ (Ocular Science, USA), and Focus ${ }^{\circledR}$ 1-2 week (Ciba Vision Corporation, USA)]. Methods: An interventional prospective clinical trial studied a sample of 40 myopic patients $(-1.00$ to $-4.50 \mathrm{sph}$, with or without astigmatism up to $-0.75 \mathrm{cyl})$. Each patient had one eye randomized to visual performance evaluation. Results: The Zywave aberrometer detected a over refraction and significant difference between Acuvue ${ }^{\circledR} 2$ and Biomedics ${ }^{\circledR} 55$ regarding spheric refractive components and spheric equivalent. Both soft contact lenses showed hypercorrection as compared to Focus ${ }^{\circledR}$ 1-2 week. Visual performance was not significantly different with spectacles and the three soft contact lenses in visual acuity and contrast sensitivity measurements. The wavefront analysis detected a significant difference in a third order aberration with and without soft contact lenses, with better visual performance with Acuvue ${ }^{\circledR} 2$ and Biomedics ${ }^{\circledR}$ 55. Conclusion: In visual performance evaluation with spectacles and soft contact lenses the wavefront analysis was a more sensible measurement of visual function 
when compared to high contrast visual acuity and contrast sensitivity. The evaluation model of visual performance with wavefront analysis developed in this investigation may be useful for further similar studies.

Keywords: Myopia; Eyeglasses; Contact lenses; Visual acuity; Contrast sensitivity; Prospective studies

\section{REFERÊNCIAS}

1. Stephens GL. Correction with single vision spectacle lenses. In: Benjamin WJ, editor. Borish's clinical refraction. Philadelphia: Saunders; c1998. p.823-82.

2. Chalita MR, Chamon W, Schor P, Freda R, Krueger R. Resultados da literatura em tratamentos personalizados com excimer laser. In: Alves MR, Chamon W, Nose W, editores. Cirurgia refrativa. Rio de Janeiro: Cultura Médica; c2003. p.353-9.

3. Applegate RA, Massof RW. Changes in the contrast sensitivity functions induced by contact lenses wear. Am J Optom Physiol. Opt. 1975;52(12):840-6.

4. Wo G, Hess R. Contrast sensitivity function and soft contact lenses. Int Contact Lens Clin. 1979;4:171-6.

5. Mitra S, Lamberts DW. Contrast sensitivity in soft contact lens wearers. Contact Intraoocular Lens Med J. 1981;7(4):315-22.

6. Kirkpatrick DL, Roggenkamp JR. Effects of soft contact lenses in contrast sensitivity. Am J Optom Physiol. 1985;62(6):407-12.
7. Cox I, Holden BA. Soft contact lenses-induced longitudinal spherical aberration and its effect on contrast sensitivity. Optom Vis Sci. 1990;67(9):679-83.

8. Wachler BS, Phillips CL, Schanzlin DJ, Krueger RR. Comparison of contrast sensitivity in different soft contact lenses and spectacles. CLAO J. 1999;25(1):48-51.

9. Wolkstein M, Atkin A, Bodis-Wollner A. Contrast sensitivity in retinal disease. Ophthalmology. 1980; 87(11):1140-9.

10. Sekuler R, Owsley C, Berenberger R. Contrast sensitivity during provoked visual impairment in multiple sclerosis. Opthalmic Physiol Opt. 1986;6(2):229-32.

11. Grey CP. Changes in contrast sensitivity when wearing low, medium, and high water content soft lenses. J Br Contact Lens Assoc. 1986;9:21-5.

12. Grey CP. Changes in contrast sensitivity during the first hour of soft contact lens wear. Am J Optom Physiol Opt. 1986;63(9):702-7.

13. Wang L, Koch DD. Ocular high-order aberrations in individuals screened for refractive surgery. J Cataract Refract Surg. 2003;29(10):1896-903.

14. Applegate RA, MarsaCk JD, Ramos R, Sarver EJ. Interaction between aberrations to improve or reduce visual performance. J Cataract Refract Surg. 2003;29(8):1487-95.

15. Oshika T, Klyce SD, Applegate RA, Howland HC, Danasoury MA. Comparison of corneal wavefront aberrations after photorefractive and laser in situ keratomileusis. Am J Ophthalmol. 1999;127(1):1-7.

16. Wang L, Misra M, Pallikaris IG, Koch DD. A comparison of a ray-tracing refractometer, autorefractor, and computerized videokeratography in measuring pseudophakic eyes. J Cataract Refract Surg. 2002;28(2):276-82.

17. Guirao A, William DR, Cox IG. Effect of rotation and translation on the expected benefit of an ideal method to correct the eye's higher-order aberrations. J Opt Soc Am A Opt Image. Sci Vis. 2001;18(5):1003-15.

18. Guirao A, Porter J, Williams DR, Cox IG. Calculated impact of higher-order monochromatic aberrations on retinal image quality in a population of human eyes. J Opt Soc Am A Opt Image Sci Vis. 2002;19(3):620-8.

\title{
Simpósio da Sociedade Brasileira de Plástica Ocular, Vias Lacrimais e Órbita
}

\author{
11 e 12 de abril de 2008 \\ São Paulo - SP \\ Teatro da Faculdade de Medicina da USP
}

\author{
INFORMAÇÕES \\ Tel. (14) 3811-6256 \\ e-mails: sartioli@fmb.unesp.br ou flaviaoft@fmb.unesp.br
}

